\title{
Prognostic value of the maximum standardized uptake value for the locoregional control in early glottic cancer
}

\author{
Donghyun Kim¹, Yongkan Ki², Jihyeon Joo², Hosang Jeon², Dahl Park', Jiho Nam¹, Wontaek Kim \\ ${ }^{1}$ Department of Radiation Oncology, Pusan National University Hospital, Pusan National University School of Medicine, Busan, Korea \\ ${ }^{2}$ Department of Radiation Oncology, Pusan National University Yangsan Hospital, Pusan National University School of Medicine, Yangsan, Korea
}

Received: May 11, 2021

Revised: September 3, 2021

Accepted: September 29, 2021

\section{Correspondence:}

Yongkan Ki

Department of Radiation Oncology,

Pusan National University Yangsan

Hospital, Pusan National University

School of Medicine, 20 Geumo-ro,

Mulgeum-eup, Yangsan 50612,

Korea.

Tel: +82-55-360-3450

E-mail:apex7171@hanmail.net

ORCID:

https://orcid.org/0000-0003-0757-8211
Purpose: To evaluate the prognostic value of the pretreatment maximum standardized uptake value (SUVmax) for locoregional control (LRC) of early glottic cancer treated with primary radiotherapy. Materials and Methods: We retrospectively reviewed the medical records of 101 patients with T1T2N0 glottic cancer treated with helical tomotherapy between 2013 and 2016. The clinical T-stages were $\mathrm{T} 1$ in 87 (86.1\%) and T2 in 14 (13.9\%) patients. The median total dose was 63 Gy (63-67.5 Gy) in 2.25 Gy per fraction. The survival outcomes were plotted using Kaplan-Meier curves. Receiver operating characteristic curves were used to assess the optimal SUVmax cut-off value for predicting locoregional recurrence.

Results: The median follow-up period was 58 months (range, 11 to 90 months). The 5 -year overall survival (OS) and locoregional recurrence-free survival rates were $96.8 \%$ and $85.4 \%$, respectively. The median pretreatment SUVmax of the primary tumor for all 101 patients was 2.3 (range, 1.1 to 9.1). The best cut-off value for SUVmax for predicting LRC was 3.3, with a sensitivity of $78.6 \%$ and specificity of $73.6 \%$. Univariate analysis showed that T-stage, overall treatment time ( $\geq 43$ days), and high SUVmax ( $\geq 3.3$ ) were significant predictors of LRC. Multivariate analysis showed that LRC was independently affected by a high SUVmax ( $\geq 3.3$ ) (hazard ratio $=5.505, p=0.020$ ).

Conclusion: High pretreatment SUVmax $(\geq 3.3)$ is a negative prognostic factor for LRC in early glottic cancer patients treated with primary radiotherapy.

Keywords: Laryngeal neoplasm, Radiotherapy, SUVmax

\section{Introduction}

High rates of local control and laryngeal preservation can be achieved with transoral laser stripping, partial laryngectomy, or radiotherapy (RT) for early glottic cancer [1]. The incidence of lymph node or distant metastases is very low in early-stage laryngeal cancers due to poor lymphatic drainage in the glottis $[2,3]$. Primary RT of the larynx without elective nodal irradiation facilitates a high cure rate and preservation of the larynx [4]. RT also provides a comparable local control rate and voice quality for T1a cases and a better cure rate for $\mathrm{T} 1 \mathrm{~b}$ cases than transoral laser excision $[5,6]$.
The outcomes of salvage partial laryngectomy after RT failure are similar to those of primary partial laryngectomy [7]. Thus, primary RT is usually prescribed for early glottic cancers to protect normal tissues.

Several prognostic factors for local control of early glottic carcinoma after definitive RT have been reported, including T-stage, anterior commissure $(\mathrm{AC})$ involvement, tumor size, dose per fraction, and overall treatment time $(0 T T)[8,9]$. Tumor bulk has been proposed as an important prognostic factor for local control of RT by several studies, but there is a limit to the objective measurement of tumor size using direct laryngoscopy or radiologic imaging studies

(C) 2021 The Korean Society for Radiation Oncology

This is an Open Access article distributed under the terms of the Creative Commons Attribution Non-Commercial License (http://creativecommons.org/licenses/by-nc/4.0/) which permits unrestricted non-commercial use, distribution, and reproduction in any medium, provided the original work is properly cited. 
$[10,11]$. There have been reports that primary tumor size has a statistically significant correlation with the maximum standardized uptake value (SUVmax) of ${ }^{18} \mathrm{~F}$-fluorodeoxyglucose positron emission tomography (FDG-PET) for non-small-cell lung and breast cancers $[12,13]$. Recently, the SUVmax of primary tumors has also been suggested as an adverse prognostic marker for laryngeal cancer $[14,15]$. Therefore, the aim of this study was to investigate the prognostic value of SUVmax for locoregional control (LRC) in early glottic cancer patients treated with primary RT.

\section{Materials and Methods}

\section{Patients and radiotherapy}

Early glottic cancer patients who received primary RT between January 2013 and December 2016 were retrospectively evaluated. The 109 patients with biopsy-proven T1-2N0M0 squamous cell carcinoma of the glottis were treated at our institution. Among them, 101 patients who underwent pretreatment FDG-PET imaging were included in this study. All patients were treated with the helical tomotherapy technique. The larynx was delineated as the clinical target volume (CTV) to include both false and true vocal cords, anterior and posterior commissures, arytenoids, and aryepiglottic folds, as well as the subglottic region. The planning target volume (PTV) was created by adding a 5- $\mathrm{mm}$ margin to the CTV in all directions, except for $3 \mathrm{~mm}$ posteriorly. A radiation dose of $63 \mathrm{~Gy}$ in $28 \mathrm{frac}-$ tions (2.25 Gy) was prescribed for PTV. Flexible fiberoptic laryngoscopy was performed weekly during RT to evaluate tumor response in all patients. Response adaptive radiation dose escalation (65.25 or $67.5 \mathrm{~Gy}$ ) was applied when a persistent gross lesion was observed during the laryngoscope examination after 56.25 Gy. This study was approved by the Institutional Review Board of Pusan National University Hospital (No. 2106-031-104). The informed consent was waived.

\section{FDG-PET study}

FDG-PET was prescribed after a confirmed pathologic diagnosis of glottic cancer. FDG-PET scans were acquired after a minimum of 4 hours of fasting. In addition, 4.0 MBq/ $\mathrm{kg}$ of FDG was administered intravenously 1 hour before imaging. Skull to mid-thigh PET images were acquired using a 3D acquisition mode at 3 minutes per bed position. The PET scans were reconstructed using computed tomography (CT) attenuation maps. The SUV was calculated as follows:

$$
\text { SUV }=\frac{\text { Measured activity concentration }(\mathrm{MBq} / \mathrm{mL})}{\text { Injected activity }(\mathrm{MBq}) / \text { Body weight }(\mathrm{g})}
$$

After all hypermetabolic tumor foci were segmented, the software calculated the SUVmax, which was defined as the highest pixel value of the SUV within the region of interest.

\section{Statistical analysis}

The primary endpoint was LRC. Locoregional recurrence-free survival (LRFS) was measured from the first day of radiotherapy to the date of histologic confirmation of local and/or regional disease recurrence. Overall survival (OS) was defined as the duration between the initiation of RT and the date of death from any cause. Receiver operating characteristic (ROC) curve analysis was used to determine the optimal cut-off value for SUVmax for LRC. The Kaplan-Meier method was used to analyze the LRC and OS rates. The log-rank test was used for univariate analysis to compare the clinical variables. The Cox proportional hazards model was used for the multivariate analysis. Statistical significance was defined as a p-value of < 0.05. SPSS version 25.0 (IBM Corp. Armonk, NY, USA) was used for all the statistical analyses.

\section{Results}

\section{Clinical characteristics}

The clinical characteristics of the patients are summarized in Table 1.

Table 1. Patient and treatment characteristics

\begin{tabular}{lc}
\hline Characteristic & $n(\%)$ \\
\hline Age (yr) & \\
$\geq 65$ & $56(55.4)$ \\
$\quad<65$ & $45(44.6)$ \\
Sex & \\
$\quad$ Male & $99(98.0)$ \\
Female & $2(2.0)$ \\
T-stage & \\
T1a & $61(60.4)$ \\
T1b & $26(25.7)$ \\
T2 & $14(13.9)$ \\
AC involvement & \\
Negative & $59(58.4)$ \\
Positive & $42(41.6)$ \\
Visible mass on CT & \\
Negative & $64(64.2)$ \\
Positive & $37(35.8)$ \\
FDG uptake (SUVmax) & \\
$<3.3$ & $67(66.3)$ \\
$\geq 3.3$ & $34(33.7)$ \\
Total dose (Gy) & \\
$<64.0$ & $64(63.4)$ \\
$\geq 64.0$ & $37(36.6)$ \\
Overall treatment time (day) & \\
$<43$ & $75(74.3)$ \\
$\geq 43$ & $26(25.7)$ \\
\hline AC, & \\
$\quad$ anterior commissure; & \\
&
\end{tabular}

$\mathrm{AC}$, anterior commissure; $\mathrm{CT}$, computed tomography; FDG, ${ }^{18} \mathrm{~F}$-fluorodeoxyglucose. 
The median age was 66 years (range, 43 to 82 years), and the median follow-up period was 58 months (range, 11 to 90 months). The clinical T stages were T1 in 87 (86.1\%) patients and T2 in 14 (13.9\%) patients. Laryngeal masses were detected on CT images of 37 patients (35.8\%), which were reconstructed with $2.0 \mathrm{~mm} \mathrm{sec-}$ tion thickness. AC involvement was present in $41.6 \%$ of patients.

Thirty-seven patients (36.6\%) received a dose above $64.0 \mathrm{~Gy}$ of irradiation, and five of them were treated with $67.5 \mathrm{~Gy} / 30$ fractions. The median 0TT was 39 days (range, 37 to 52 days); 75 patients (74.3\%) completed treatment within 43 days. The two non-compliant patients were treated for a long period (50 and 52 days, respectively). They frequently missed treatment fractions voluntarily and received 65.25 Gy in 29 fractions, resulting in a prolonged 0 Tा.

The median pretreatment SUVmax of the primary tumor for all 101 patients was 2.3 (range, 1.1 to 9.1). ROC curve analysis showed that the area under the curve (AUC) for LRC was 0.785 (95\% confidence interval, 0.673-0.897; $p=0.001$ ). The best cut-off value of SUVmax was 3.3 , and the sensitivity and specificity were $78.6 \%$ and $73.6 \%$, respectively (Fig. 1). Thirty-four patients (33.7\%) had a high SUVmax ( $\geq 3.3$ ). The median duration between laryngeal biopsy and PET scanning was 12 days (range, 1 to 35 days). There was no significant correlation between the biopsy interval to PET and SUVmax ( $p=0.797)$, and FDG uptake was not affected by the laryngeal biopsy.

\section{Survival analysis}

The 5-year OS and LRFS rates were 96.9\% and 85.4\%, respectively (Fig. 2). Four patients expired during the follow-up. Only one patient died of recurrent glottic cancer 13 months after treatment.
The causes of the deaths of the other three patients were secondary primary colon cancer (one patient) and pneumonia (two patients), respectively, during a period of no evidence of laryngeal cancer. Disease failure was observed in 14 patients (13.9\%), and all relapses were confirmed by biopsy. Thirteen patients (12.9\%) experienced local recurrence, and one patient had a regional lymph node recurrence without local failure. After recurrence, they underwent salvage surgery including 10 total laryngectomies, two laser cordectomies, one supracricoid partial laryngectomy for local recurrence, and one modified radical neck dissection for regional re-

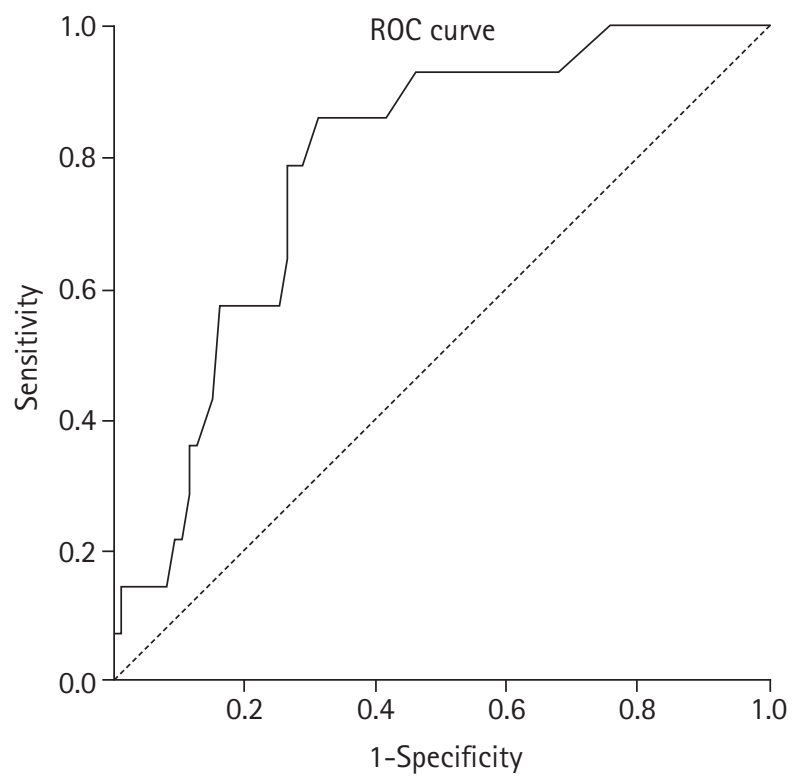

Fig. 1. Receiver operating characteristic (ROC) curve for the relationship of pretreatment SUVmax with the locoregional control.
(A)

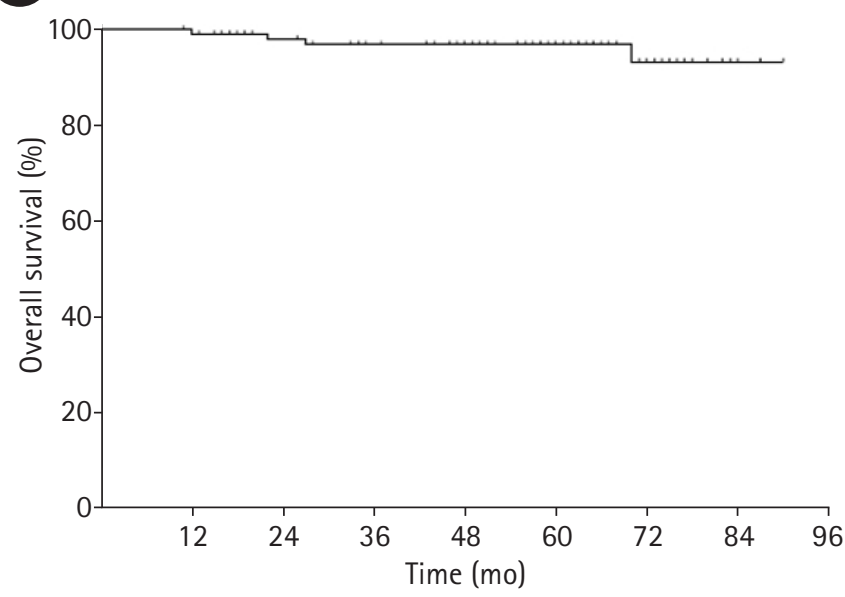

B

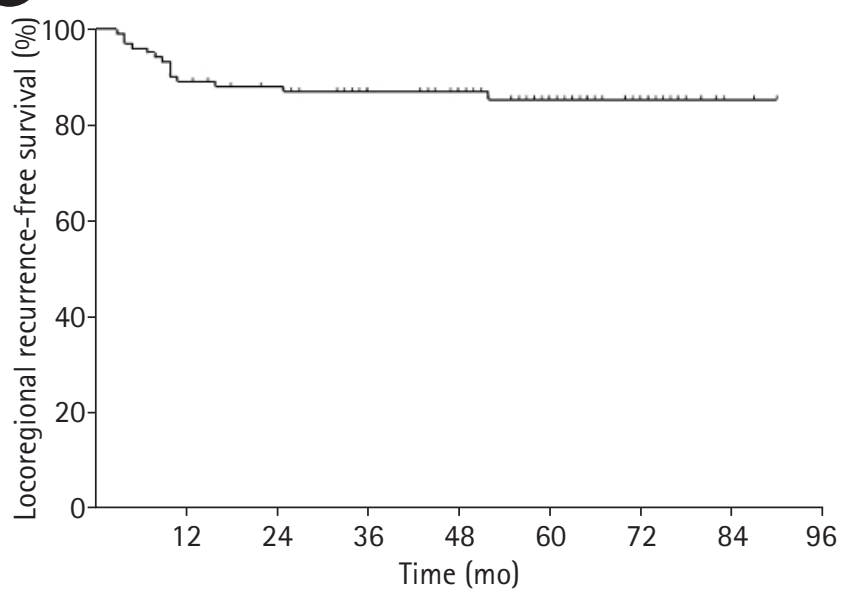

Fig. 2. (A) Overall survival curve and (B) locoregional recurrence-free survival curves. 
currence. Twelve patients (85.7\%) were alive and had no evidence of disease after salvage treatment.

The mean LRFS time was 86.5 months for patients with low SUVmax $(<3.3)$ and 62.0 months for patients with high SUVmax $(\geq 3.3)$ ( $p<0.001)$. Univariate analysis revealed that the significant prognostic factors for LRC were clinical T-stage ( $p=0.005)$, high SUVmax ( $\geq 3.3$ ) ( $<<0.001)$, and $0 \pi$ ( $\geq 43$ days) ( $p=0.004)$. The Kaplan-Meier survival curves for these prognostic factors are shown in Fig. 3. The visible mass on CT images and total treatment dose had marginal correlation with $\operatorname{LRC}(p=0.081$ and $p=0.091$, respectively). Multivariate analysis showed that high SUVmax $(\geq 3.3$ ) was an independent negative prognostic factor (hazard ratio $=5.505, p=0.020$ ) (Table 2).

T-stage was the only significant prognostic factor in the univariate analysis for overall survival ( $p=0.032$ ). The 5 -year OS rates were $97.4 \%$ and $92.9 \%$ for patients with $\mathrm{T} 1$ and $\mathrm{T} 2$ disease, respectively (Fig. 4A). There was no significant difference in OS between the two groups: patients with SUVmax of $\geq 3.3$ and those with SUVmax of $<3.3$ ( $p=0.829$ ) (Fig. 4B).

\section{Discussion and Conclusion}

This study evaluated the prognostic value of the SUVmax of glottic lesions for local control in early glottic cancer. Pretreatment SUVmax was the only significant independent prognostic factor for recurrence in RT for early glottic cancer ( $p<0.001$ ) (Fig. 3B). This is consistent with a report that showed that SUVmax of $>3.4$ was a significant prognostic factor in patients with T1 and T2 glottic cancers treated by RT [14]. The ${ }^{18} \mathrm{~F}-\mathrm{FDG}-\mathrm{PET}$ scan was analyzed to determine its prognostic value for various head and neck cancer subtypes. A previous study reported that PET imaging has no clinical benefit for laryngeal cancer [16]; however, several previous reports have shown that high FDG uptake (SUVmax >3.4-10.0) is significantly associated with local recurrence in head and neck cancers $[14,17,18]$. The results of this study suggest that a higher SUVmax is associated with a more aggressive nature and lower RT response rate [17]. Kitajima et al. [18] showed that a high nodal SUVmax $(\geq 4.0)$ was a significant factor for progression-free survival (PFS) in patients with laryngeal cancer, including advanced stages treated with RT with or without chemotherapy. They demonstrated that the high primary tumor SUVmax was not correlated with PFS.

The tumor bulk has been considered an important tumor-related prognostic factor in laryngeal cancer and has been proposed to be included in the current staging systems for laryngeal cancer $[19,20]$. A previous study reported that disease recurrence increased dramatically up to $26 \%$ when the laryngeal mass size was more than $15 \mathrm{~mm}$ [21]. However, there is concern about the reli-
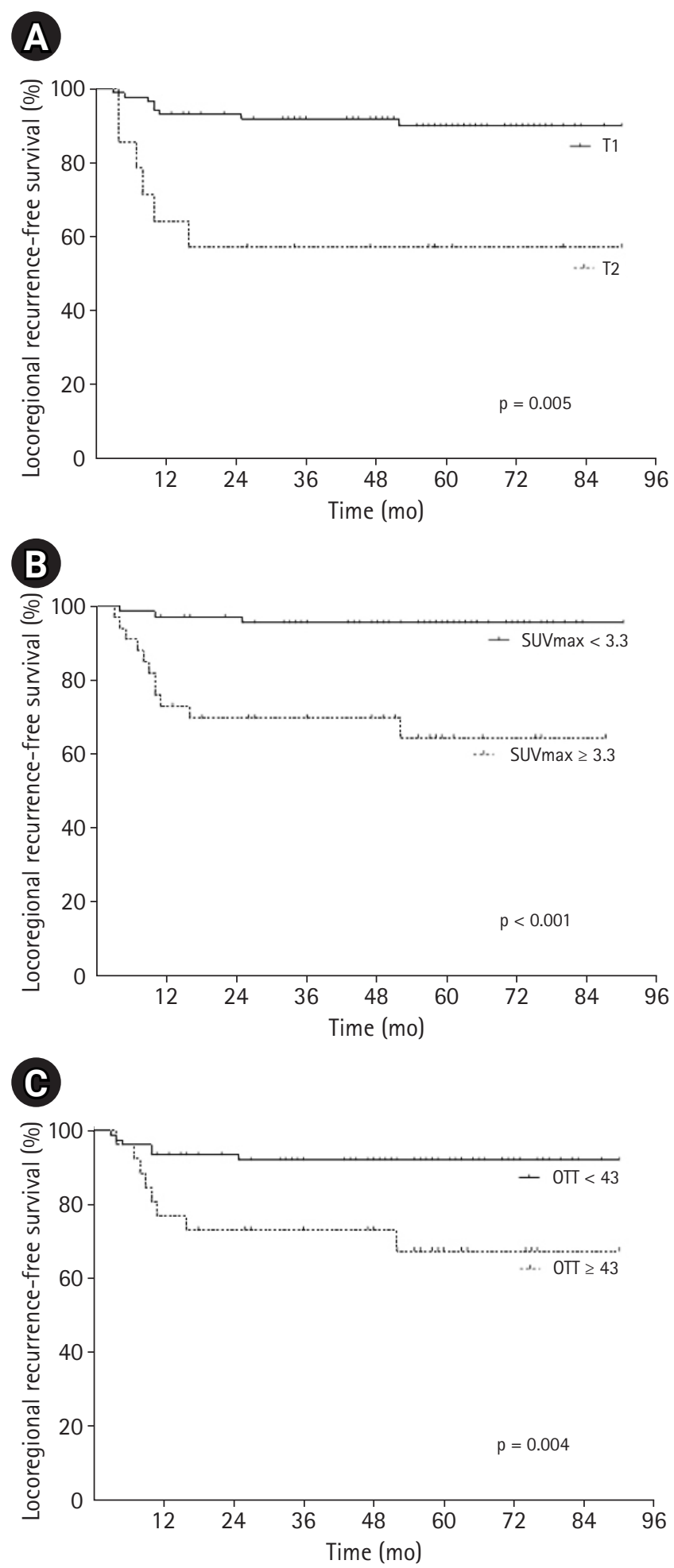

Fig. 3. Locoregional recurrence-free survival curves according to (A) T-stage, (B) pretreatment SUVmax of ${ }^{18} \mathrm{~F}$-fluorodeoxyglucose positron emission tomography (FDG-PET) and (C) overall treatment time (OTT).

ability of objectively measuring tumor volume. Several reports have shown significant intra- and interobserver variabilities in laryngeal 
Table 2. Univariate and multivariate analysis of factors associated with locoregional recurrence-free survival

\begin{tabular}{|c|c|c|c|c|}
\hline \multirow{2}{*}{ Variable } & \multicolumn{2}{|c|}{ Univariate } & \multicolumn{2}{|c|}{ Multivariate } \\
\hline & 5 -yr rate $(\%)$ & $p$-value & $\mathrm{HR}(95 \% \mathrm{Cl})$ & $p$-value \\
\hline \multicolumn{5}{|l|}{ Age (yr) } \\
\hline$<65$ & 83.3 & 0.712 & - & \\
\hline$\geq 65$ & 87.3 & & - & \\
\hline \multicolumn{5}{|l|}{ Sex } \\
\hline Male & 85.0 & 0.570 & - & \\
\hline Female & 100 & & - & \\
\hline \multicolumn{5}{|l|}{ T-stage } \\
\hline $\mathrm{T} 1$ & 88.7 & $0.005^{*}$ & $1.858(0.586-5.890)$ & 0.292 \\
\hline $\mathrm{T} 2$ & 64.3 & & - & \\
\hline \multicolumn{5}{|c|}{ AC involvement } \\
\hline Negative & 88.7 & 0.190 & - & \\
\hline Positive & 81.0 & & - & \\
\hline \multicolumn{5}{|c|}{ Visible mass on $\mathrm{CT}$} \\
\hline Negative & 90.5 & 0.081 & - & \\
\hline Positive & 76.2 & & - & \\
\hline \multicolumn{5}{|c|}{ FDG uptake (SUVmax) } \\
\hline$<3.3$ & 95.4 & $<0.001^{*}$ & $5.505(1.315-23.054)$ & $0.020^{*}$ \\
\hline$\geq 3.3$ & 65.4 & & - & \\
\hline \multicolumn{5}{|c|}{ Total dose (Gy) } \\
\hline$<64$ & 90.4 & 0.091 & - & \\
\hline$\geq 64$ & 76.5 & & - & \\
\hline \multicolumn{5}{|c|}{ Overall treatment time (day) } \\
\hline$<43$ & 91.9 & $0.004^{*}$ & $1.819(0.573-5.776)$ & 0.310 \\
\hline$\geq 43$ & 67.5 & & - & \\
\hline
\end{tabular}

$\mathrm{AC}$, anterior commissure; $\mathrm{CT}$, computed tomography; $\mathrm{FDG}$, ${ }^{18} \mathrm{~F}$-fluorodeoxyglucose; $\mathrm{HR}$, hazard ratio; $\mathrm{Cl}$, confidence interval. ${ }^{*} \mathrm{p}<0.05$.
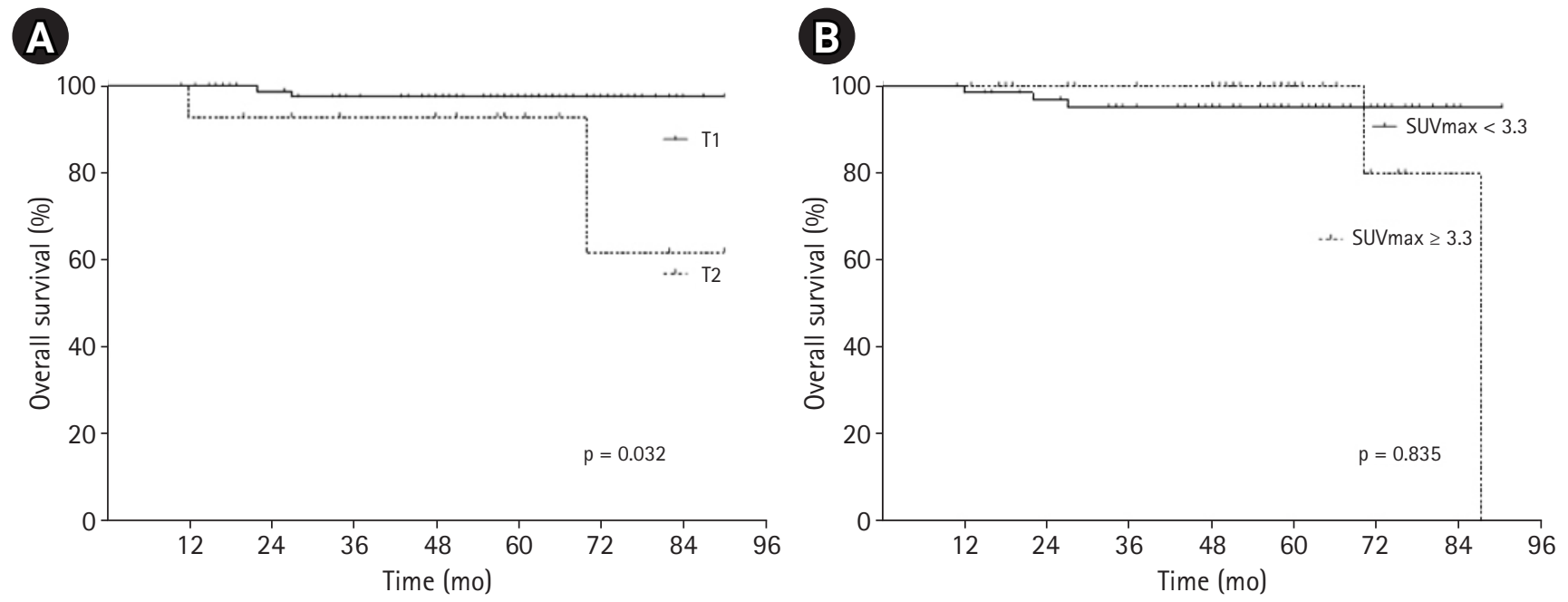

Fig. 4. Overall survival curves according to (A) T-stage and (B) pretreatment SUVmax of ${ }^{18} \mathrm{~F}$-fluorodeoxyglucose positron emission tomography (FDG-PET).

tumor volume measurements based on CT images [22,23]. They suggested that reliable tumor volume measurements could be obtained by a single trained reader. There are still limitations related to reproducibility across the measurements by various observers from different institutions. In our study, laryngeal masses observed on CT images did not help predict the outcome of RT ( $p=0.081)$. 
Univariate analysis showed a significant correlation between the T-stage and local control ( $p=0.005)$; however, multivariate analysis showed no significant prognostic value $(p=0.292)$. Of the 14 patients with T2 stages, 10 (71.4\%) had a greater SUVmax ( $\geq 3.3)$, which showed that SUVmax rather than the T-stage was correlated with lower tumor control rates in these patients. Based on an evaluation of a larger number of T2 stage patients, the T-stage may have independent prognostic values for LRC. There was a significant correlation between T-stage and OS, but only one patient with T2 stage died of recurrent cancer. A much longer follow-up is required for better reliability on this result.

Patients with an 0T of 43 days or more also showed significantly lower LRC rates in the univariate analysis ( $p=0.004)$. This is in agreement with previous reports $[9,24]$. A shorter 0TT using hypofractionation with 2.25 Gy per fraction was associated with higher local control rates [9]. In the present study, all patients were treated with a daily fraction size of 2.25 Gy. Multivariate analysis showed no significant correlation between the $0 \Pi$ and DFS ( $p=0.310)$.

A higher total dose showed a borderline significant association with a lower $L R C$ rate in the univariate analysis ( $p=0.091$ ). $\mathrm{Pa}-$ tients with a lower radiation response of the tumor were prescribed a higher radiation dose because we increased the prescription dose above 65 Gy when the gross lesion had remained until the end of treatment. There was a significant correlation between a higher SUVmax and a higher radiation dose ( $p=0.002)$. Of the 34 patients with higher SUVmax ( $\geq 3.3), 20$ (58.8\%) required higher radiation doses. On the other hand, only 14 of 67 patients (20.9\%) with lower SUVmax (<3.3) received doses above $65 \mathrm{~Gy}$.

Anterior commissure infiltration was reported to be an adverse prognostic factor for early glottic cancer [25]. However, there are various conflicting reports on this $[26,27]$. AC is considered a weak point for tumor spread because of the lack of thyroid cartilage perichondrium to resist tumor invasion [28]. Another suggestion is that the air-tissue interface of AC is associated with the risk of underdosing, which may lead to poor coverage by the prescribed dose [29]. However, the negative impact of AC involvement could be overcome by delivering a fraction dose of more than $2.0 \mathrm{~Gy}$ [30]. Our study that used 2.25 Gy per fraction also showed no significant relationship between AC invasion and LRC ( $p=0.190)$.

This study was limited by its retrospective design performed at a single institution and the varied time intervals between the laryngeal biopsy and PET scan. Further prospective controlled studies with more patients are needed to confirm the results of this study. Nevertheless, our results suggest that a high SUVmax ( $\geq 3.3)$ in patients with early glottic cancer treated with primary RT showed a lower disease control rate. Therefore, alternative treatment strate- gies such as concurrent chemoradiotherapy should be considered for these patients.

\section{Conflict of Interest}

No potential conflict of interest relevant to this article was reported.

\section{Acknowledgements}

This work was supported by a 2-Year Research Grant of Pusan National University.

\section{References}

1. Mendenhall WM, Werning JW, Hinerman RW, Amdur RJ, Villaret DB. Management of T1-T2 glottic carcinomas. Cancer 2004; 100:1786-92.

2. Skladowski K, Tarnawski R, Maciejewski B, Wygoda A, Slosarek K. Clinical radiobiology of glottic T1 squamous cell carcinoma. Int J Radiat Oncol Biol Phys 1999;43:101-6.

3. Warde $P$, O'Sullivan B, Bristow RG, et al. T1/T2 glottic cancer managed by external beam radiotherapy: the influence of pretreatment hemoglobin on local control. Int J Radiat Oncol Biol Phys 1998:41:347-53.

4. Lim YJ, Wu HG, Kwon TK, et al. Long-term outcome of definitive radiotherapy for early glottic cancer: prognostic factors and patterns of local failure. Cancer Res Treat 2015;47:862-70.

5. O'Hara J, Markey A, Homer JJ. Transoral laser surgery versus radiotherapy for tumour stage 1a or $1 \mathrm{~b}$ glottic squamous cell carcinoma: systematic review of local control outcomes. J Laryngol Otol 2013;127:732-8.

6. Gioacchini FM, Tulli M, Kaleci S, Bondi S, Bussi M, Re M. Therapeutic modalities and oncologic outcomes in the treatment of T1b glottic squamous cell carcinoma: a systematic review. Eur Arch Otorhinolaryngol 2017;274:4091-102.

7. Rosier JF, Gregoire V, Counoy H, et al. Comparison of external radiotherapy, laser microsurgery and partial laryngectomy for the treatment of T1NOM0 glottic carcinomas: a retrospective evaluation. Radiother Oncol 1998;48:175-83.

8. Akine Y, Tokita N, Ogino T, et al. Radiotherapy of T1 glottic cancer with 6 MeV X rays. Int J Radiat Oncol Biol Phys 1991;20:1215-8.

9. Yamazaki H, Nishiyama K, Tanaka E, Koizumi M, Chatani M. Radiotherapy for early glottic carcinoma (T1NOM0): results of prospective randomized study of radiation fraction size and overall treatment time. Int J Radiat Oncol Biol Phys 2006;64:77-82.

10. Reddy SP, Hong RL, Nagda S, Emami B. Effect of tumor bulk on 
local control and survival of patients with T1 glottic cancer: a 30-year experience. Int J Radiat Oncol Biol Phys 2007;69:138994.

11. Jin J, Liao Z, Gao L, Huang X, Xu G. Analysis of prognostic factors for $\mathrm{T}(1) \mathrm{N}(0) \mathrm{M}(0)$ glottic cancer treated with definitive radiotherapy alone: experience of the cancer hospital of Peking Union Medical College and the Chinese Academy Of Medical Sciences. Int J Radiat Oncol Biol Phys 2002;54:471-8.

12. Stiles BM, Nasar A, Mirza F, et al. Ratio of positron emission tomography uptake to tumor size in surgically resected non-small cell lung cancer. Ann Thorac Surg 2013;95:397-403.

13. Heudel P, Cimarelli S, Montella A, Bouteille C, Mognetti T. Value of PET-FDG in primary breast cancer based on histopathological and immunohistochemical prognostic factors. Int J Clin Oncol 2010;15:588-93.

14. Park JW, Lee SW, Kim JS, Song SY. Prediction of local control in early glottic carcinoma using the maximum standardised uptake value. Cancer Radiother 2017;21:205-9.

15. Werner J, Hullner MW, Rupp NJ, et al. Predictive value of pretherapeutic maximum standardized uptake value (Suvmax) in laryngeal and hypopharyngeal cancer. Sci Rep 2019;9:8972.

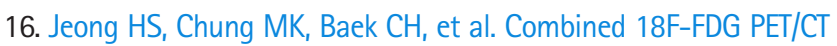
imaging for the initial evaluation of glottic cancer. Clin Exp Otorhinolaryngol 2008;1:35-40.

17. Halfpenny W, Hain SF, Biassoni L, Maisey MN, Sherman JA, McGurk M. FDG-PET: a possible prognostic factor in head and neck cancer. Br J Cancer 2002;86:512-6.

18. Kitajima K, Suenaga Y, Kanda T, et al. Prognostic value of FDG PET imaging in patients with laryngeal cancer. PLoS One 2014;9: e96999.

19. Rutkowski T. The role of tumor volume in radiotherapy of patients with head and neck cancer. Radiat Oncol 2014;9:23.

20. Issa MR, Samuels SE, Bellile E, Shalabi FL, Eisbruch A, Wolf G. Tumor volumes and prognosis in laryngeal cancer. Cancers (Basel) 2015;7:2236-61.

21. Mendenhall WM, Parsons JT, Stringer SP, Cassisi NJ, Million RR.
T1-T2 vocal cord carcinoma: a basis for comparing the results of radiotherapy and surgery. Head Neck Surg 1988;10:373-7.

22. Hermans $R$, Feron $M$, Bellon $E$, Dupont $P$, Van den Bogaert $W$, Baert AL. Laryngeal tumor volume measurements determined with CT: a study on intra- and interobserver variability. Int J Radiat Oncol Biol Phys 1998;40:553-7.

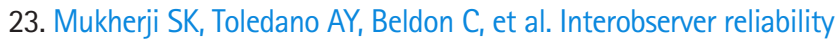
of computed tomography-derived primary tumor volume measurement in patients with supraglottic carcinoma. Cancer 2005; 103:2616-22.

24. Le QT, Fu KK, Kroll S, et al. Influence of fraction size, total dose, and overall time on local control of T1-T2 glottic carcinoma. Int J Radiat Oncol Biol Phys 1997;39:115-26.

25. Olszewski SJ, Vaeth JM, Green JP, Schroeder AF, Chauser B. The influence of field size, treatment modality, commissure involvement and histology in the treatment of early vocal cord cancer with irradiation. Int J Radiat Oncol Biol Phys 1985;11:1333-7.

26. Kim TG, Ahn YC, Nam HR, et al. Definitive radiation therapy for early glottic cancer: experience of two fractionation schedules. Clin Exp Otorhinolaryngol 2012;5:94-100.

27. Moon $\mathrm{SH}$, Cho KH, Chung E, et al. A prospective randomized trial comparing hypofractionation with conventional fractionation radiotherapy for T1-2 glottic squamous cell carcinomas: results of a Korean Radiation Oncology Group (KROG-0201) study. Radiother Oncol 2014;110:98-103.

28. Bagatella F, Bignardi L. Morphological study of the laryngeal anterior commissure with regard to the spread of cancer. Acta Otolaryngol 1981;92:167-71.

29. Bradley PJ, Rinaldo A, Suarez $C_{1}$ et al. Primary treatment of the anterior vocal commissure squamous carcinoma. Eur Arch Otorhinolaryngol 2006;263:879-88.

30. Tong CC, Au KH, Ngan RK, et al. Impact and relationship of anterior commissure and time-dose factor on the local control of T1N0 glottic cancer treated by 6 MV photons. Radiat Oncol 2011; $6: 53$. 